Proceedings of the ASME 2021 48th Annual Review of Progress in Quantitive Nondestructive Evaluation QNDE2021

July 28-30, 2021, Virtual, Online

QNDE2021/74694

\title{
REMOTE, VOLUMETRIC ULTRASONIC IMAGING OF DEFECTS USING TWO-DIMENSIONAL LASER INDUCED PHASED ARRAYS
}

\author{
Peter Lukacs, Geo Davis, Theodosia Stratoudaki,, Yashar Javadi, Gareth Pierce, Anthony Gachagan \\ Centre for Ultrasonic Engineering \\ Department of Electronic \& Electrical Engineering \\ University of Strathclyde \\ Glasgow, UK \\ Email: t.stratoudaki@strath.ac.uk
}

\begin{abstract}
Manufacturing processes, such as welding and additive manufacturing, take place at high temperatures and extreme environments that offer significant challenges to conventional nondestructive testing methods. Laser Induced Phased Arrays (LIPAs) have evolved as a promising testing method for the aforesaid applications due to its remote and couplant free operation. Contrary to transducer-based phased arrays, LIPAs are synthesized in post-processing by scanning the generation and detection lasers. The data from one-dimensional (1D) phased arrays are used to produce two-dimensional (2D), cross-sectional images, whereas the data from two-dimensional phased arrays generate three-dimensional (3D) images, thus providing more information on defect characterization. In this work, two-dimensional (2D) LIPAs are synthesized in the non-destructive thermoelastic regime using lasers for ultrasonic generation and detection, in order to image defects at different depths inside an aluminum sample. The acquired data is processed using the Total Focusing Method (TFM) algorithm to obtain volumetric images representing the interior of the sample. A $3 D$ finite element $(F E)$ model is also developed to support the experiments.
\end{abstract}

\footnotetext{
*Address all correspondence to this author.
}

\section{INTRODUCTION}

Ultrasonic imaging is a useful tool in analyzing the integrity of components non-destructively. Ultrasonic transduction can be achieved using piezoelectric transducers, electromagnetic acoustic transducers (EMATs), and laser excitation. Piezoelectric transducers and EMATs are commonly employed in the industry due to their ease of use and cost effectiveness. With industries now moving to online process monitoring to reduce wastage and carbon footprint, there is a demand for techniques to be applied in extreme environments. However, piezoelectric transducers are not designed to withstand the high temperatures offered by manufacturing processes such as welding and additive manufacturing [1]. Laser ultrasonics (LU) is a broadband, remote and couplant free technique that can be employed for complex geometries, hostile environments and places of restricted access [2]. However, LU generates signals with a low signal-to-noise ratio (SNR) [3]. The SNR can be improved by focusing and steering the ultrasonic beam (beamforming), which is the case in ultrasonic phased arrays. In conventional, transducer based arrays, the beamforming action results from the use of multiple array elements. However, implementing multiple lasers for LU is not viable due to the high hardware demand.

In response, Laser Induced Phased Arrays (LIPAs) have been developed, based on principles of LU. In LIPAs, a phased array is synthesized using the superposition theorem [4]. A signal is captured for each combination of generation and detection 
element position, by scanning a single generation and a single detection laser, following the paradigm of the Full Matrix Capture (FMC) [5]. The advantage of capturing the Full Matrix is that it can be post processed in a range of algorithms. Cross-sectional imaging using 1D LIPAs have been successfully demonstrated on additively manufactured components created using Wire+Arc Additive Manufacturing (WAAM) [6] and Selective Laser Melting [7], detecting cylindrical defects with a diameter of $0.25 \mathrm{~mm}$ in the latter case.

The topic of the presented study is 3D, volumetric imaging using $2 \mathrm{D}$ phased arrays. The advantage of this type of imaging is that it provides a means of volumetric inspection in situations where access to the specimen is restricted, without the need for mechanical probe manipulators. Additionally, real defects and structures are three dimensional such as in the case of cracking and inclusions in welds and can occur in arbitrary orientations. 2D arrays can generate volumetric images for better defect characterization than that can be achieved with a $1 \mathrm{D}$ array, due to the wider range of inspection angles [8,9].

In this work, we synthesize a 2D LIPA in order to achieve remote, 3D imaging using LU for the first time. Until now, the restrictions have been the limited number of elements previously achieved and the slow speed of data collection. These challenges are addressed in the presented study. The FMC from the array was processed using the total focusing method algorithm to generate a volumetric image of the interior of the sample. We also present the experimental and numerical results for 2D LIPA for the case of Selective Matrix Capture.

\section{BACKGROUND}

This section gives a brief overview on the directivity and sensitivity patterns of the generated ultrasound as a consequence of the laser-material interaction and on the total focusing method algorithm implemented for generating the ultrasonic images.

\section{Ultrasonic Directivity and Sensitivity}

In LU, ultrasound is generated and detected using lasers [3]. In the thermoelastic generation, the shear wave is more strongly excited than the longitudinal [10] and has maximum amplitude at around $\pm 30^{\circ}$ [11]. Similarly, depending upon the component of displacement (out-of-plane/in-plane) there exists a sensitivity pattern for the detection method.

Figure 1(a) gives the directivity pattern of the shear wave based on analytical expressions for laser generated ultrasound, at the non-destructive, thermoelastic regime, in aluminum [4]. Figure 1(b) gives the sensitivity pattern based on analytical expressions for the out-of-plane displacements for the shear wave in aluminum, as this was the ultrasonic component detected by the detection interferometer used in this study [4].

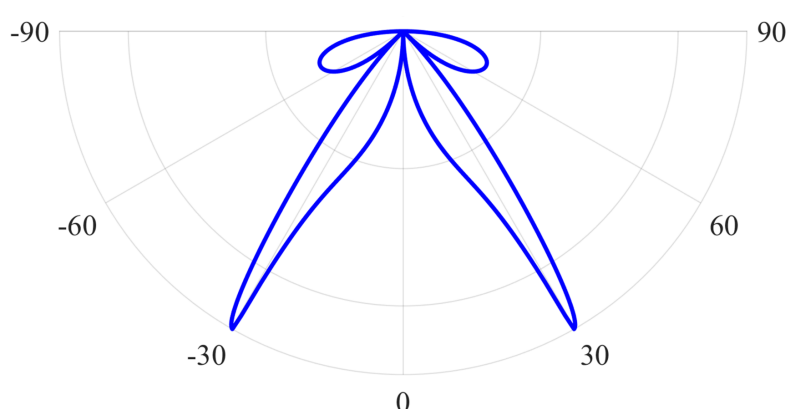

(a)

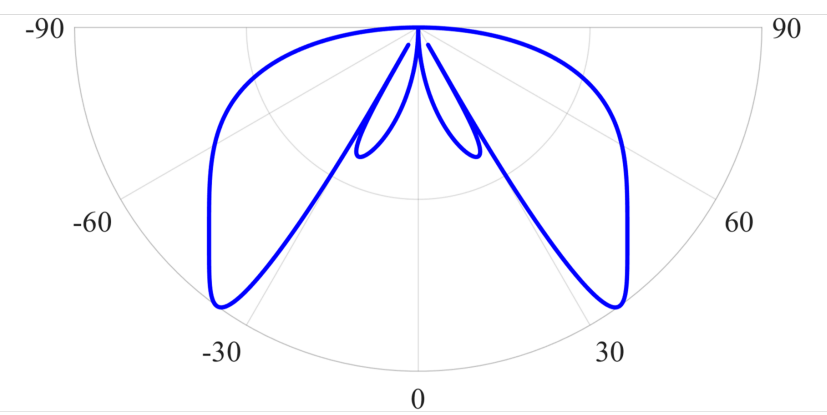

(b)

FIGURE 1. DIAGRAM OF (a) LASER GENERATED ULTRASOUND DIRECTIVITY PATTERN AND (b) SENSITIVITY PATTERN OF OUT-OF-PLANE COMPONENT DETECTOR FOR SHEAR WAVES IN ALUMINUM AS A FUNCTION OF ANGLE.

\section{Total Focusing Method}

The imaging algorithm used in this study was the Total Focusing Method (TFM): dynamic focusing is achieved on a userdefined grid by time shifting the A-scan signals. The delay is calculated using the ray paths to and from each pixel of the grid [5]. As focusing is achieved on every pixel, in TFM the images generated have superior image quality compared to that of previous techniques such as focused B-scan or sectoral scan.

\section{FINITE ELEMENT SIMULATION}

A 3D finite element model was developed using COMSOL Multiphysics ${ }^{\circledR}$ to support the experiments. The laser pulse (100 ns) was modelled as a transient surface heat flux. The sample size considered was $2 \times 2 \times 2 \mathrm{~mm}$ with a $1 \mathrm{~mm}$ diameter cylindrical defect, placed at $1 \mathrm{~mm}$ from the scan surface (see fig. 2). For meshing the geometry, the mesh element size was decided based on two regions of interest, namely, the thermal wave region and the ultrasonic wave region [12]. The thermal wave region is modelled using smaller mesh elements as compared to the ultrasonic wave region. In the ultrasonic wave region, one-tenth of 


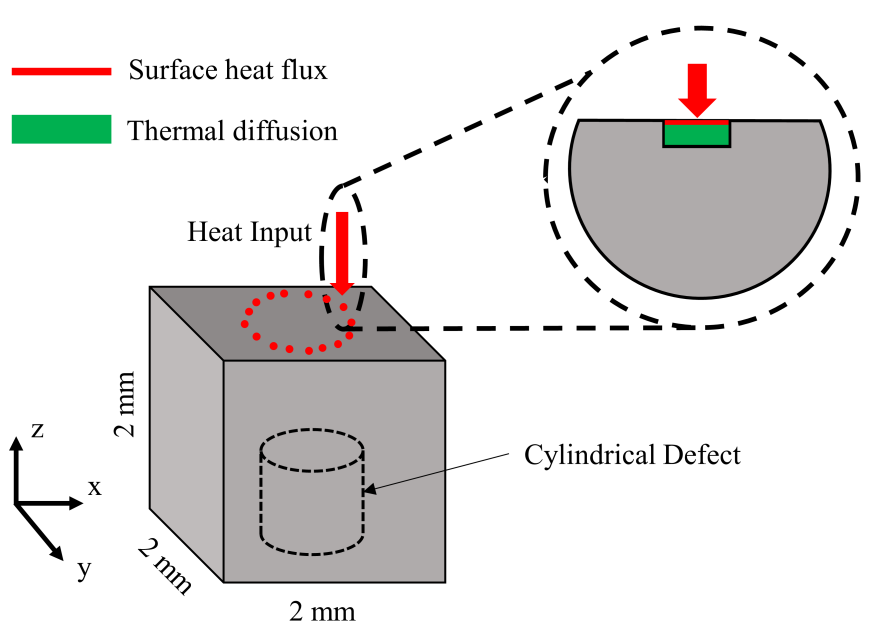

(a)

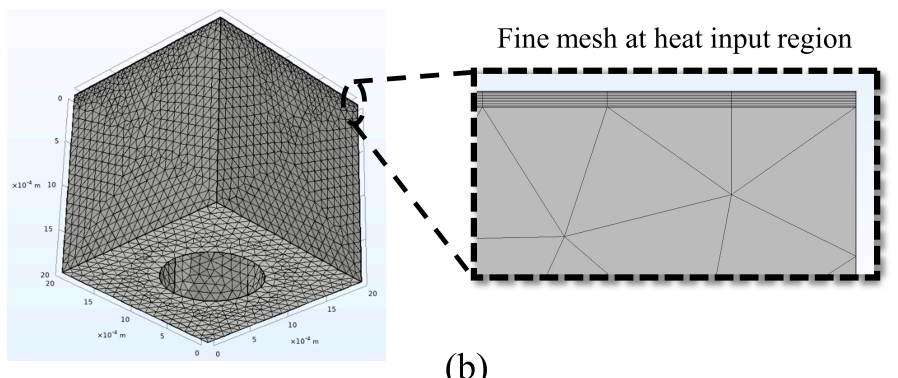

(b)

FIGURE 2. (a) 3D MODEL SHOWING THE GEOMETRY OF THE PART CONSIDERED WITH A CYLINDRICAL DEFECT OF $1 \mathrm{MM}$ DIAMETER AT THE CENTER AND THE POSITION OF THE ARRAY ELEMENTS, REPRESENTED BY THE RED DOTS. (b) THE MESH STRATEGY IMPLEMENTED FOR THE MODEL SHOWING FINER MESHES FOR THE HEAT INPUT REGION.

the smallest ultrasonic wavelength is considered for maximum mesh size. The time step considered for the simulation was 10 ns for a total simulation time of $2 \mu \mathrm{s}$.

\section{EXPERIMENTAL METHODS}

This section gives details on the sample and experimental setup used in this study. Information on the imaging algorithms used is also given briefly.

\section{Experimental Sample}

The sample used is shown shown in fig. 3. It was an aluminum sample with dimensions of $49 \times 90 \times 20 \mathrm{~mm}$. Various defects were present on the sample, however for the case of this study the focus was on three of the $1 \mathrm{~mm}$ diameter, cylindrical, flat-bottom blind holes made using Electrical Discharge Machining. These defects were at varying depths, at 5, 10 and $15 \mathrm{~mm}$

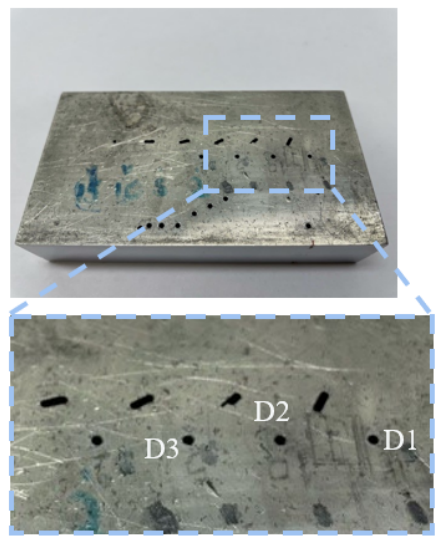

(a)

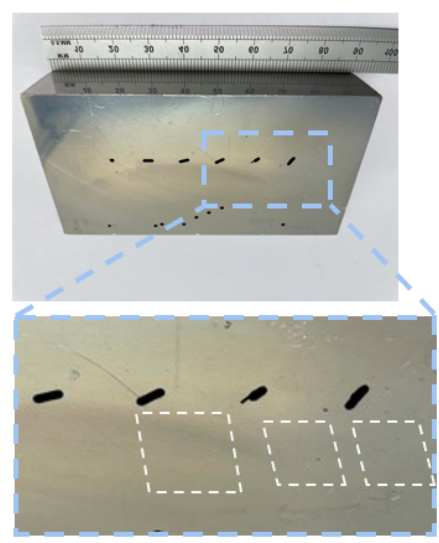

(b)

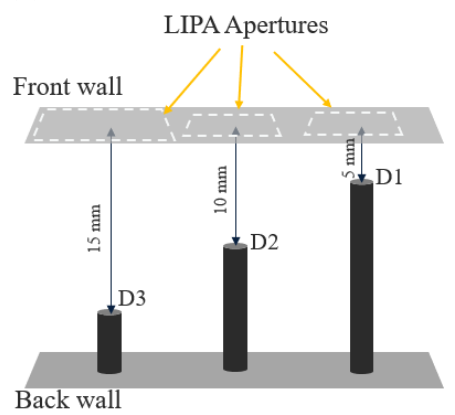

(c)

FIGURE 3. (a) PHOTO OF TEST SAMPLE'S BACK WALL AND (b) SCAN-SURFACE. (c) A DIAGRAM OF THE SAMPLE'S INTERIOR. DEFECTS D1-D3 ARE INDICATED ON THE BACK WALL. THE WHITE DASHED LINES SHOW THE LOCATION OF THE 3 LIPA APERTURES.

from the scanned surface. The surface finish on the inspection side was polished to a smooth finish in order to provide better return for the detection laser, improving SNR.

\section{Experimental Setup}

The experimental setup can be seen on fig 4. Detection of ultrasound waves was performed using a rough surface interferometer (Quartet - Sound \& Bright) that had a bandwidth of 1$66 \mathrm{MHz}$ and was measuring the out-of-plane component of the acoustic field. The detector laser had a power of $780 \mathrm{~mW}, 532$ $\mathrm{nm}$ wavelength and was placed $200 \mathrm{~mm}$ from the sample's scan surface. The detector's laser-head was scanned in two dimensions, horizontally and vertically, using two scanning stages (M511.DD1, Physik Instrumente \& KMTS50E/M, Thorlabs).

For ultrasonic generation, a Q-switched, Nd:YAG laser (Elforlight) was used, with a pulse width of $8 \mathrm{~ns}$, average power of $600 \mathrm{~mW}$ and a repetition rate of $1 \mathrm{kHz}$. The output of the laser was focused onto a spot on the sample's surface using a spherical 


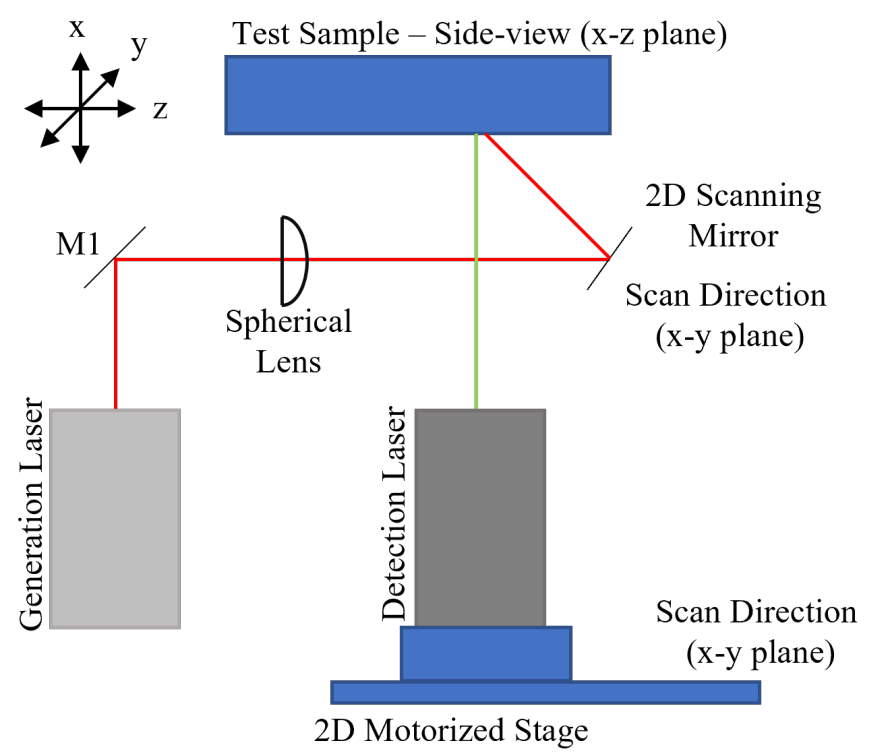

FIGURE 4. EXPERIMENTAL SETUP USED FOR SYNTHESIS OF 2D LIPA.

lens. The position of the generation point was controlled using a dual scanning galvanometer mirror (GVS302, Thorlabs), for vertical and horizontal movement. The scanning mirror was operated using a DAQ unit (USB-6001, National Instrument) and all the equipment were controlled using Labview.

\section{Imaging Algorithms}

The imaging algorithm used in this study was the Total Focusing Method (TFM), which is a delay-and-sum algorithm that time-shifts and combines signals appropriately for each pixel achieving dynamic focusing everywhere on the predefined image [5]. As the laser generated ultrasound has a broadband spectrum -nominally from DC to $100 \mathrm{MHz}$, digital filtering can be performed before data analysis, allowing for adjustment of the inspection frequency in post-processing. Digital filtering was performed using a Gaussian filter, with $100 \%$ bandwidth before applying TFM on the data sets.

During post-processing a Vector Coherence Factor (VCF) was introduced to the imaging algorithm in order to improve the image quality [13]. VCF is a variation of Phase Coherence Imaging (PCI) [13] and measures the coherence of phase throughout the A scan signals for any given pixel/voxel. In this way, the effects of grating lobes and random noise can be significantly reduced, as their contribution to any pixel will be incoherent, leading to low phase-coherence [14]. The benefits of PCI are especially important for volumetric imaging, as this can allow for extending inter-element spacing beyond the Nyquist sampling limit of half the acoustic wavelength [15]. By distributing elements in
TABLE 1. SYNTHESIZED LIPA PARAMETERS

\begin{tabular}{lcccc}
\hline & Elements & Pitch & Filtering & Averaging \\
\hline LIPA 1 & $11 \times 11$ & $0.6 \mathrm{~mm}$ & $6 \mathrm{MHz}$ & 32 \\
LIPA 2 & $11 \times 11$ & $0.6 \mathrm{~mm}$ & $6 \mathrm{MHz}$ & 32 \\
LIPA 3 & $11 \times 11$ & $0.8 \mathrm{~mm}$ & $3.5 \mathrm{MHz}$ & 32 \\
\hline
\end{tabular}

2 dimensions, dense array apertures are spatially limited, however this limit is overcome by the supression of grating lobes by PCI [14].

\section{RESULTS}

This section describes the data acquisition methods used for experimental and simulated experiments and compares results.

\section{Full Matrix Capture}

During data acquisition three dense, 2D LIPAs were synthesized with a total of 100 elements, in a dense matrix layout with equidistant pitch in both dimensions. The LIPAs above defects D1 and D2 had the same pitch and aperture, however for the case of D3 the LIPA aperture had to be expanded in order to achieve better sensitivity deeper within the sample. The LIPA parameters can be seen in Table 1 along with the digital filtering center frequency used in post-processing. For LIPAs $1 \& 2$, a $6 \mathrm{MHz}$ center frequency was use, to achieve better resolution, however as D3 was deeper from the scan surface, lower frequency contents were utilized, which were centered around $3.5 \mathrm{MHz}$, to account for losses due to attenuation. Using the acquired data sets, 3 images were produced using the shear wave mode, one for each defect (fig. 5). The defects' depth can be observed relative to the scan surface at 5,10 and $15 \mathrm{~mm}$ depth.

\section{Selective Matrix Capture Acquisition}

In the previous section, results from 2D LIPAs with a dense matrix array layout - a commonly used 2D phased array design were shown. The following section shows the results when creating an optimized design called Selective Matrix Capture, which adapts the array parameters to the defect and the characteristics of the laser ultrasound inspection method [16]. The process was performed both in simulation and in post-processing, using one of the previously acquired data sets.

Finite Element Results This section gives the finite element results for a 24-element LIPA, simulating the SMC method of data acquisition. The array elements are optimized for 


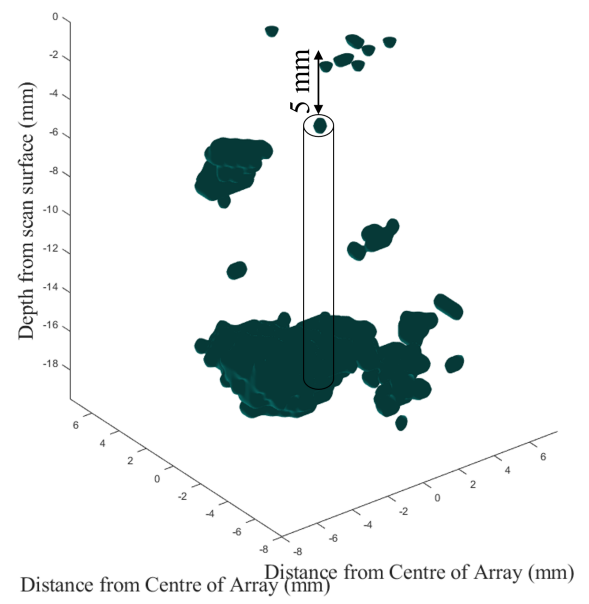

(a)

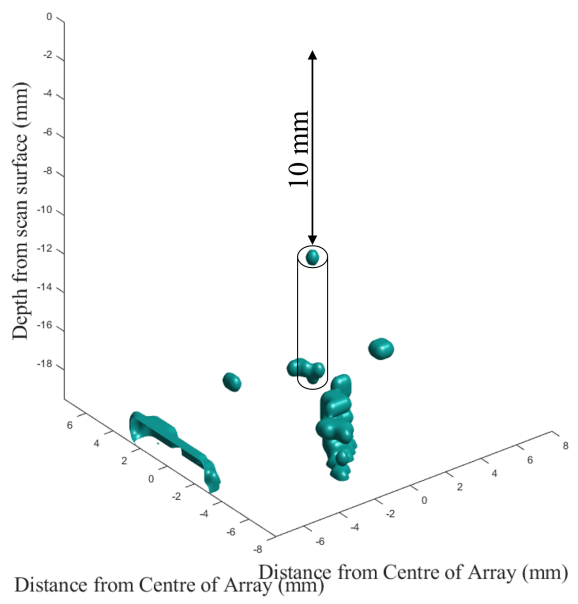

(b)

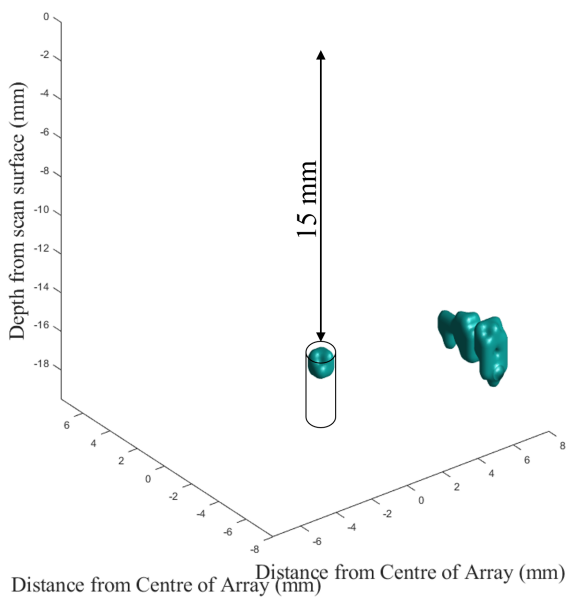

(c)

FIGURE 5. VOLUMETRIC, EXPERIMENTAL TFM IMAGES OF SHEAR WAVES FROM 2D LIPAs. (a) DEFECT D1, (b) DEFECT D2 AND (c) DEFECT D3.

maximum sensitivity based on the sensitivity maps [17]. Out-ofplane displacements are recorded and processed using the TFM algorithm. The image in fig. 6 is obtained by processing the shear waves, with a bandpass filter of $6 \mathrm{MHz}$ center frequency. The feature in the image is located at $1 \mathrm{~mm}$ from the scan surface and is indicative of the circular face of the cylindrical defect. The measured diameter of the cylindrical defect is $0.96 \mathrm{~mm}$ as compared to the actual diameter of $1 \mathrm{~mm}$.

Experimental Results For the case of the experimental results, SMC was emulated by removing elements of the previously captured Full Matrix data set that only contributed to noise and did not contain any useful information. The resultant array comprised of 40 elements, selected for their location with respect to the defect location: each selected element was at an angle of optimal sensitivity (i.e. around 30 degrees for aluminum) based on the laser ultrasonic directivity and sensitivity patterns, as shown in the background section (fig. 1). The resulting image can be seen on fig. 7(b).

\section{DISCUSSION}

In this study the first volumetric images using 2D Laser Induced Phased Arrays are presented. In fig. 5 the defects are identified at the expected location and depth in the sample.

The proposed SMC data acquisition method synthesizes a flexible, adaptive LIPA that has the capabilities to optimize array parameters to the needs of inspection, on-the-fly. In this work this array flexibility was utilized to change the aperture size in order to have better visibility at larger depth in the sample. This was performed without adjusting the experimental setup. In con-

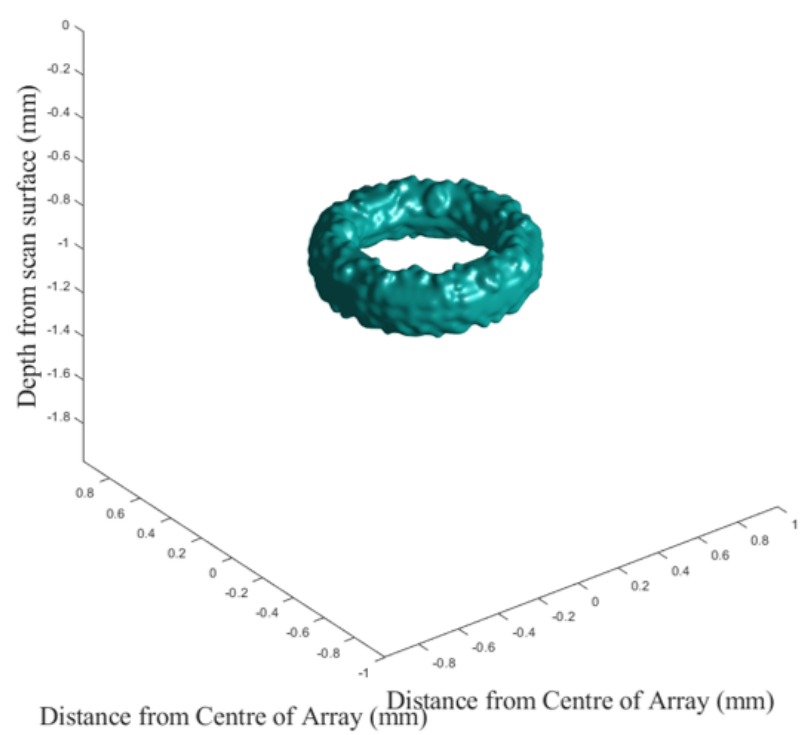

FIGURE 6. VOLUMETRIC IMAGE PRODUCED USING FINITE ELEMENT ANALYSIS OF A 1 MM DIAMETER FLAT BOTTOM HOLE AT 1 MM DEPTH. GAUSSIAN DIGITAL FILTERING WAS APPLIED TO THE A SCAN SIGNALS AT 6 MHZ WITH 100\% BANDWIDTH.

trast, this would not have been possible using the 3D imaging performed by conventional, transducer-based phased array. For future work, the adaptability of the LIPA can be further utilized to change additional characteristics, such as inter-element spacing and layout. For this first demonstration of 2D LIPAs, a dense 


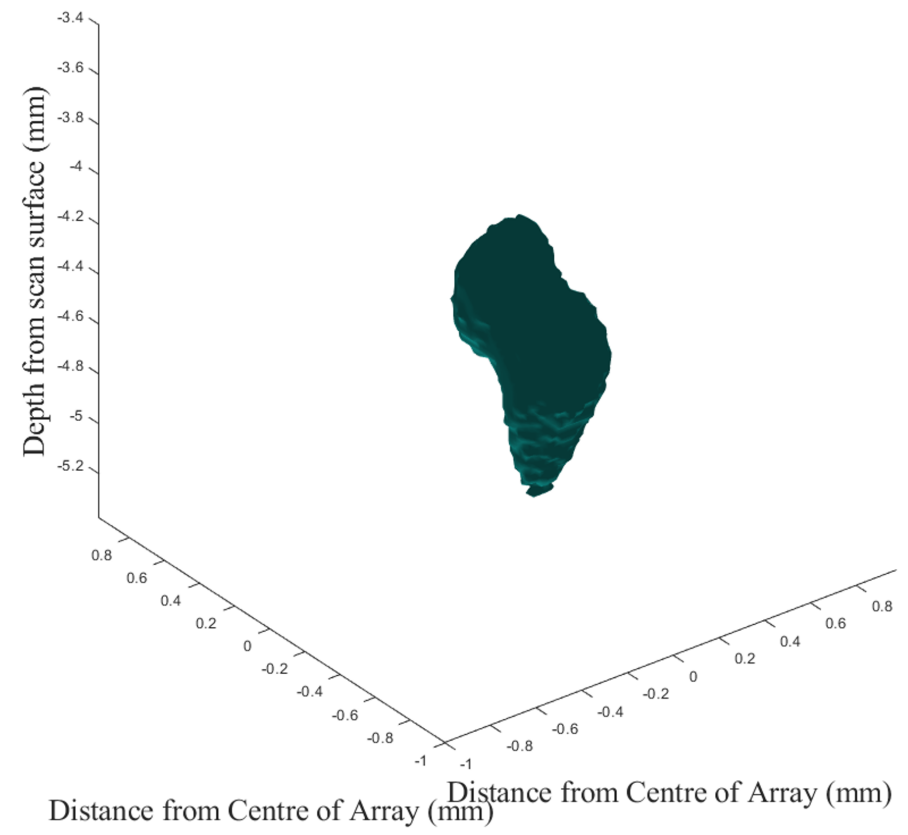

(a)

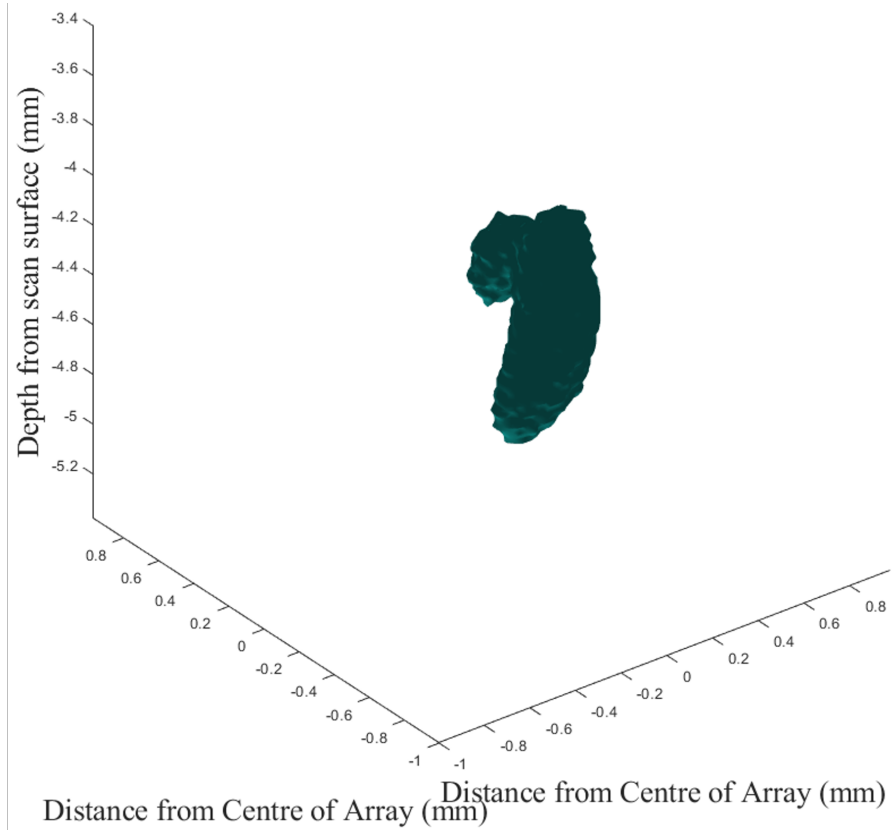

(b)

FIGURE 7. CLOSE UP EXPERIMENTAL TFM IMAGES OF SHEAR WAVES SHOWING DEFECT D1 AT DEPTH OF 5 MM, USING: (a) FULL MATRIX CAPTURE WITH LIPA OF 100 ARRAY ELEMENTS AND (b) SELECTIVE MATRIX CAPTURE WITH LIPA OF 40 ARRAY ELEMENTS.

matrix layout was used, however for future work a wide range of array designs will be used, combined with SMC, that will significantly improve imaging quality and data acquisition time, using the same experimental setup.

Regarding SMC, it is possible to produce 2D arrays that are optimized for the internal features of the sample. This optimized array would address a critical challenge of 2D phased arrays: data size and data acquisition time. In order to compare the two data acquisition methods, FMC and SMC, the quality of the two images were analyzed. Figure 7 shows close up images of defect D1 with an image size of $2 \times 3 \times 3 \mathrm{~mm}$, centered around the defect. While the two images aren't identical, an overall good agreement can be observed between the two shapes and their sizes. More importantly, an improvement of nearly 10 times was achieved for data acquisition time by only synthesizing the 40 array elements that contribute useful information out of the total 100 elements of the FMC. This translates to a reduction in data acquisition time from 40 minutes, in the case of FMC, to around 4.5 mins for SMC. Furthermore, an improvement of data size was also observed from $55 \mathrm{MB}$ (FMC) to $6 \mathrm{MB}$ (SMC).

The flexibility offered from LIPAs in terms of array characteristics, such as element number, location and pitch, can be used for rapid prototyping of any array design, allowing for experimental testing while adjusting array parameters. This feature of LIPAs holds a significant potential for array designing and prototyping, potentially leading to faster and more accurate experimental assessment of array designs.

\section{SUMMARY}

2D LIPAs of 11 x 11 elements were implemented. Flatbottom blind holes were successfully imaged to a maximum depth of $15 \mathrm{~mm}$ from the scanned surface, using the acquired data set, on an aluminum sample. Additionally, a 3D finite element model was developed to simulate 2D LIPAs. The results from the simulation and the experimental data set were used to demonstrate the SMC data acquisition method for volumetric imaging. The SMC would increase the speed of inspection by nearly 10 times faster than that of the commonly used FMC while still imaging the defect efficiently. In addition, 9 times reduction of data volume was achieved.

In future work, the SMC method will be implemented using 2D LIPAs during the data acquisition stage rather than in post-processing. Volumetric imaging on rough surfaces such as welded and as-deposited, additively manufactured surfaces will further establish the versatility of the technique and its application potential. Furthermore, LIPAs will be extended to inspect complex defects and components with curved surfaces. 


\section{ACKNOWLEDGMENT}

This work was supported by the UK Engineering and Physical Sciences Research Council [EP/T012862/1, EP/R513349/1]. P. Lukacs was supported by a EPSRC PhD studentship [EP/R513349/1]. The authors wish to thank Dr Charles MacLeod of the EEE department, University of Strathclyde, Glasgow, for supporting the research by providing computing resources and software for finite element modeling.

\section{REFERENCES}

[1] Vithanage, R. K. W., Mohseni, E., Qiu, Z., MacLeod, C., Javadi, Y., Sweeney, N., Pierce, G., and Gachagan, A., 2020. "A phased array ultrasound roller probe for automated in-process/interpass inspection of multipass welds". IEEE Transactions on Industrial Electronics, pp. 1-1.

[2] Davies, S. J., Edwards, C., Taylor, G. S., and Palmer, S. B., 1993. "Laser generated ultrasound: its properties, mechanisms and multifarious applications". J. Phys. D: Appl. Phys., 26, pp. 329-348.

[3] Scruby, C. B., and Drain, L. E., 1990. Laser Ultrasonics, Techniques and Applications. Adam Hilger, Bristol, UK.

[4] Stratoudaki, T., Clark, M., and Wilcox, P. D., 2016. "Laser induced ultrasonic phased array using full matrix capture data acquisition and total focusing method". Opt. Express, 24(19), pp. 21921-21938.

[5] Holmes, C., Drinkwater, B. W., and Wilcox, P. D., 2005. "Post-processing of the full matrix of ultrasonic transmit-receive array data for non-destructive evaluation". NDT \& E International, 38, p. 701-711.

[6] Lukacs, P., Davis, G., Stratoudaki, T., Williams, S., MacLeod, C. N., and Gachagan, A., 2021. "Remote ultrasonic imaging of a wire arc additive manufactured ti$6 \mathrm{ai}-4 \mathrm{v}$ component using laser induced phased array". In 2021 IEEE International Instrumentation and Measurement Technology Conference (I2MTC), pp. 1-6.

[7] Pieris, D., Stratoudaki, T., Javadi, Y., Lukacs, P., Catchpole-Smith, S., Wilcox, P. D., Clare, A., and Clark, M., 2020. "Laser induced phased arrays (lipa) to detect nested features in additively manufactured components". Materials \& Design, 187, p. 108412.

[8] Velichko, A., and Wilcox, P. D., 2011. "Defect characterization using two-dimensional arrays". In Review of Progress in Quantitative Nondestructive Evaluation, Thompson, DO and Chimenti, DE, ed., Vol. 1335 of AIP Conference Proceedings, pp. 835-842.

[9] McKee, J. G., Bevan, R. L. T., Wilcox, P. D., and Malkin, R. E., 2020. "Volumetric imaging through a doubly-curved surface using a 2d phased array". NDT \& E International, 113, p. 102260.

[10] Rose, L. R. F., 1984. "Point-source representation for laser- generated ultrasound". J. Acoust. Soc. Am., 75, pp. 723732.

[11] Noroy, M.-H., Royer, D., and Fink, M., 1993. “The lasergenerated ultrasonic phased array: Analysis and experiments". J. Acoust. Soc. Am., 94, pp. 1934-1943.

[12] Davis, G., Balasubramaniam, K., Palanisamy, S., Nagarajah, R., and Rajagopal, P., 2020. "Additively manufactured integrated slit mask for laser ultrasonic guided wave inspection”. Int. J. Adv. Manuf. Technol., 110, pp. 1203-1217.

[13] Camacho, J., Fritsch, C., Fernandez-Cruza, J., and Parrilla, M., 2019. "Phase coherence imaging: Principles, applications and current developments". Proceedings of Meetings on Acoustics, 38(1), p. 055012.

[14] Camacho, J., Parrilla, M., and Fritsch, C., 2009. "Phase coherence imaging”. IEEE Trans. Ultrason., Ferroelectr., Freq. Control, 56(5), pp. 958-974.

[15] Wooh, S. C., and Shi, Y., 1999. "Optimum beam steering of linear phased arrays". Wave Motion, 29(3), pp. 245-265.

[16] Lukacs, P., Stratoudaki, T., Wilcox, P., Clark, M., and Gachagan, A., 2019. "Optimisation of data acquisition and processing for laser induced ultrasonic phased arrays". In Proceedings of Meetings on Acoustics, Vol. 38, p. 030015.

[17] Stratoudaki, T., Clark, M., and Wilcox, P. D., 2017. "Adapting the full matrix capture and the total focusing method to laser ultrasonics for remote non destructive testing". In 2017 IEEE International Ultrasonics Symposium (IUS), pp. $1-4$. 